\title{
Quantum Chromodynamics and
}

\section{Nuclear Physics at Extreme Energy Density}

\author{
Progress Report
}

May 15, 1994 - May 14, 1995

\author{
BERNDT MÜLLER \\ ROXANNE P. SPRINGER \\ Department of Physics \\ Duke University \\ Durham, NC 27708-0305
}

15 May 1995

MISTERTOH of The DCOMENT IS UNLMTED

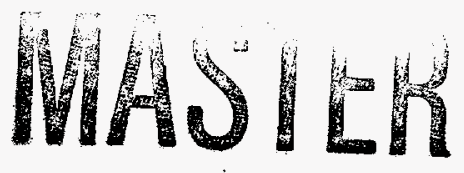

\footnotetext{
Prepared for the U.S. Department of ENERgy UNDER GRANT NUMBeR DE-FG05-90ER40592
} 


\section{DISCLAIMER}

Portions of this document may be illegible in electronic image products. Images are produced from the best available original document. 


\section{DISCLAIMER}

This report was prepared as an account of work sponsored by an agency of the United States Government. Neither the United States Government nor any agency thereof, nor any of their employees, make any warranty, express or implied, or assumes any legal liability or responsibility for the accuracy, completeness, or usefulness of any information, apparatus, product, or process disclosed, or represents that its use would not infringe privately owned rights. Reference herein to any specific commercial product, process, or service by trade name, trademark, manufacturer, or otherwise does not necessarily constitute or imply its endorsement, recommendation, or favoring by the United States Government or any agency thereof. The views and opinions of authors expressed herein do not necessarily state or reflect those of the United States Government or any agency thereof. 


\section{Contents}

1 Quark-Gluon Plasma and Relativistic Heavy Ion Collisions 3

1.1 Open Charm Production in an Equilibrating Parton Plasma . . . . . . . . 3

1.2 Physics and Signatures of the Quark-Gluon Plasma . . . . . . . . . . . 5

1.3 Quark Pair Production in the Chiral Transition . . . . . . . . . . 6

1.4 Distillation of Strangelets for Low Initial $\mu / T \ldots \ldots \ldots$

$1.5 \mathrm{DCC}$ and Strong Electromagnetic Fields . . . . . . . . . . . . 10

1.6 Lyapunov Exponent and Plasmon Damping Rate . . . . . . . . . . . 12

1.7 Dynamics of Soft Modes in Thermal Gauge Theories . . . . . . . . . . 13

1.8 Collisions of Yang-Mills Wavepackets . . . . . . . . . . . . . 14

1.9 Interface Tension and Entropy . . . . . . . . . . . . . . . . 15

2 Effective Theories for Hadrons and Nuclei 16

2.1 Kaon-Nucleon-Nucleon Couplings for Decays of Hypernuclei . . . . . . . 16

2.2 Limits on Possible T-Violating, P-Conserving Observables . . . . . . . . . 18

2.3 Baryon Magnetic Moments from a Chiral Extrapolation of Lattice Results. . 19

2.4 Photoproduction of $\pi^{0}$ on Light Nuclei . . . . . . . . . . . . 20

3 Other Topics 22

3.1 Renormalization Group Approach to Field Theory at Finite Temperature . . 22

3.2 Symmetry-Preserving Regularization . . . . . . . . . . . . . . 24

3.3 An Effective Field Theory Approach to the Cosmological Constant Problem 25

$\begin{array}{lr}\text { A Publications } & \mathbf{2 7}\end{array}$

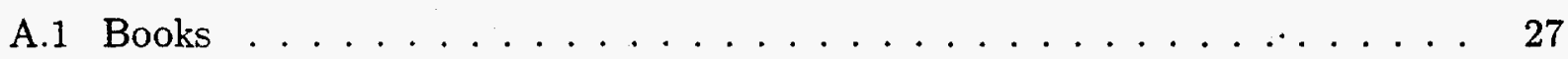

A.2 Published Articles . . . . . . . . . . . . . . . . . . 27

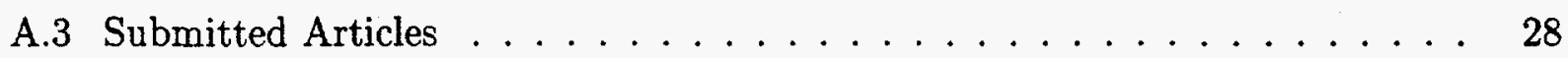

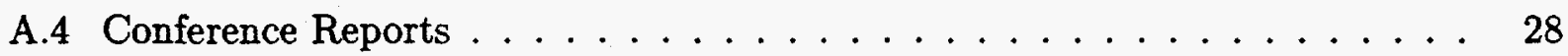

B Lecture Activities $\quad \mathbf{3 0}$

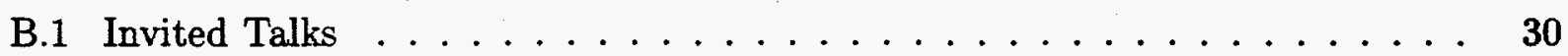

B.2 Contributed Talks and Seminars . . . . . . . . . . . . . 31

$\begin{array}{ll}\text { C Triangle Nuclear Colloquium Series } & 34\end{array}$

$\begin{array}{ll}\text { D Theory Seminars at Duke } & 36\end{array}$ 


\title{
1 Quark-Gluon Plasma and Relativistic Heavy Ion Collisions
}

\subsection{Open Charm Production in an Equilibrating Parton Plasma}

\author{
B. Müller
}

At extremely high energies, nucleus-nucleus collisions may be described by parton interactions in the framework of QCD-inspired models. In this framework, hard or semihard scatterings among partons dominate the reaction dynamics. They can liberate partons from the individual confining nucleons, producing a large amount of transverse energy in the central rapidity region and eventually driving the parton system toward equilibrium. The same hard processes, such as open charm pair production, direct photon and dilepton emission, can also be used as direct probes of the early parton dynamics and the early evolution of the quark-gluon plasma.

Unlike strange quarks, charm quarks cannot be easily produced during the mixed and hadronic phases of the dense matter since the $c$ quark mass is much larger than the corresponding temperature scale. The only period when charm quarks can be easily produced is during the early stage of the parton evolution when the effective temperature is still high. At this stage, the parton gas is still not fully equilibrated so that the temperature is only an effective parameter describing the average momentum scale. By measuring this preequilibrium charm production, one can probe the initial parton density in phase space and shed light on the equilibration time [1].

Charm production can be divided into three different contributions in the history of the evolution of the parton system: (1) initial production during the nuclear overlap period ; (2) pre-thermal production from secondary parton scatterings during the thermalization, $\tau<$ $\tau_{\text {iso }}$; and (3) thermal production during the parton equilibration, $\tau>\tau_{\text {iso }}$, in the expanding system. We have used the results of the HIJING Monte Carlo model [2] to estimate the initial parton production. For stage (3) we approximate the resulting parton distributions by locally equilibrated phase space densities and assume that elastic parton scattering is sufficiently rapid to maintain local thermal equilibrium during the expansion. However, we treat chemical equilibration of the parton plasma explicitly using the master equations derived in [3].

With the given evolution of the parton gas, we can calculate open charm production during the parton equilibration. Similar to light quarks, charm quarks are produced through gluon fusion $g g \rightarrow c \bar{c}$ and quark antiquark annihilation $q \bar{q} \rightarrow c \bar{c}$ during the evolution of the parton plasma. To model the phase-space distribution before the local parton distributions reach isotropy in momentum space, we take into account the distribution of the initial production points which spread over a region of width $\Delta_{k} \approx \frac{2}{k_{1} \cosh y}$ in the direction of the beam axis, where $y$ is the c.m. rapidity of the partons. We assume free-streaming until $\tau_{\text {iso }}$ and neglect the expansion in the transverse direction. The correlated phase-space 
distribution function is given by

$$
f(k, x)=\frac{1}{g_{G} \pi R_{A}^{2}} g\left(k_{\perp}, y\right) \frac{e^{-(z-t \tanh y)^{2} / 2 \Delta_{k}^{2}}}{\sqrt{2 \pi} \Delta_{k}} \theta\left(R_{A}-r\right) \theta\left(\tau_{\mathrm{iso}} \cosh y-t\right),
$$

where $g\left(k_{\perp}, y\right)$ is the parametrization of the parton spectrum given by HIJING simulations. The correlation between momentum and space-time was not considered in our previous calculation [1]. It turns out that this correlation is very important and reduces the prethermal charm production significantly.

Plotted in Figure 1 as dot-dashed and dashed lines are the pre-thermal and thermal production. In the calculation, a $k$-factor of two multiplies the lowest order matrix elements of charm production. We find that both the thermal contribution during the parton equilibration and pre-thermal contribution with the current estimate of the initial parton density from HIJING Monte Carlo simulation are much smaller than the initial direct charm production. However, the final total charm production is very sensitive to the initial condition of the parton evolution. If the initial parton production were higher by a factor of 4 , resulting in a fully equilibrated parton plasma, the total secondary charm yield would become comparable or larger than the initial production, due to both the increase production rate and longer life time of the parton plasma. We also found that open charm production is more sensitive to the initial temperature of the parton system than to the initial parton fugacities. Therefore, open charm production is a good probe of the initial parton distribution in phase space and the thermalizaion and equilibration time of the parton plasma.

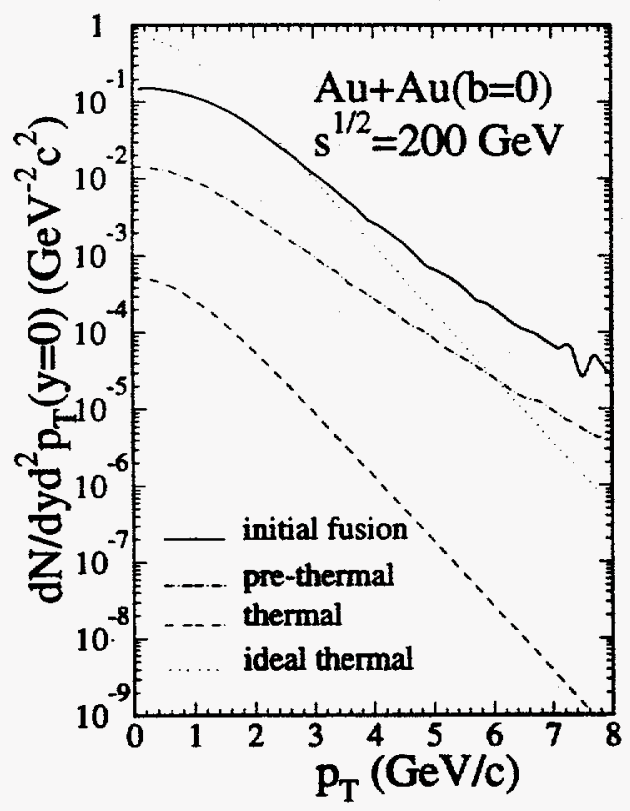

Figure 1: The $p_{\perp}$ distributions of initial (solid), prethermal (dot-dashed), and thermal (dashed) charm production for central $\mathrm{Au}+\mathrm{Au}$ collisions at RHIC energy, $\sqrt{s}=200 \mathrm{AGeV}$. The dotted line is the thermal production assuming an initial fully equilibrated QGP at the same temperature.

1. B. Müller and X.-N. Wang, Phys. Rev. Lett. 68, 2437 (1992). 
2. X.-N. Wang and M. Gyulassy, Phys. Rev. D44, 3501 (1991).

3. T. S. Biró, E. van Doorn, B. Müller, M. H. Thoma, and X.-N. Wang, Phys. Rev. C48, 1275 (1993).

\subsection{Physics and Signatures of the Quark-Gluon Plasma}

\section{B. Müller}

This review [1] of the current status of theoretical studies on the most promising quarkgluon plasma signals groups the proposed quark-gluon plasma signatures into five categories according to the different physical properties of superdense hadronic matter which they probe. These are: (1) thermodynamic variables measuring the equation of state; (2) probes for chiral symmetry restoration; (3) probes of the colour response function; (4) probes of the electromagnetic response function; and (5) "exotic" signatures of the quark-gluon plasma. The review begins with a brief survey of our current picture of the dynamical and structural properties of the quark gluon plasma, of its creation and evolution, then proceeds to the analysis of the various proposed signals and concludes with an assessment of their merits and ambiguities.

The experimental detection of a quark-gluon plasma in relativistic heavy ion reactions at collider energies requires a combination of signals which probe different aspects of the hightemperature phase of QCD. It is first necessary to establish the main reaction mechanism, to obtain experimental information on the initial conditions, and to determine the lifetime of the hot, dense fireball. Electromagnetic probes, charm yield, and density interferometry are promising tools for this purpose. It would be especially interesting to demonstrate the preponderance of gluonic degrees of freedom in the initial entropy production, as predicted by parton cascade models. If the thermalization times are as short, and the initial densities as high as currently predicted, the observation of a state consisting of quasifree quarks and gluons should be possible with little ambiguity.

The existence of a rather long-lived mixed phase at the quark-hadron phase transition should be visible in flow effects and in the lepton-pair spectrum. Strange baryons are an excellent probe of dense baryon-rich matter. The observed enhancements are perhaps the best indication, so far, that hadronic reaction models are insufficient. The observation of disoriented chiral domains would clearly demonstrate the presence of the chiral phase transition. Metastable strangelets, if produced and found, would provide unassailable evidence for the existence of quark matter.

An important goal of relativistic heavy ion physics is the exploration of the reaction mechanism at high energies. Is there a transition from the low energy regime, where reactions can be successfully described as interacting hadronic cascades, to a high-energy regime, where quark and gluon constituents provide a much simpler description of the first few $\mathrm{fm} / c$ of the reaction? Do the pomeron-dominated soft strong interactions disappear at sufficiently high energy, giving way to bulk interactions that can be much more economically described in the framework of perturbative QCD? An experimental demonstration of these features would 
constitute, in itself a major step toward, the discovery of the quark-gluon plasma. The fact that hadronic cascade models are beginning to fail and become inconsistent at the highest currently accessible energies provides reason for optimism that this transition may be near.

1. B. Müller, Rep. Prog. Phys. 28, 1 (1995).

\subsection{Quark Pair Production in the Chiral Transition}

\section{Greiner [1]}

In ultrarelativistic heavy ion collisions it is expected that chiral symmetry is temporarily restored. As already reported last year, we have begun to explore the consequences that would ensue if the restoration of the initially broken chiral vacuum happens on a very short time scale in the course of the collision. If the underlying QCD vacuum changes its structure rather suddenly, the transition is accompanied by a spontaneous and nonperturbative production of quark pairs. In the current quark picture the 'hadronic' vacuum state $\mid 0$ In $\rangle$ corresponds to a complex coherent superposition of current quark states which are allowed to go rapidly out of phase. Occupied states of the negative Dirac continuum are partially excited into the open positive continuum. This can be calculated by solving for the Dirac wavefunctions in the time dependent scalar background field [2]. In Figure 1 the spectrum of particles produced in a 'smooth' transition are shown for various, finite choices of the total duration of the transition $\Delta t \approx 1.5 \tau$. The constituent quark mass was set to $350 \mathrm{MeV}$, while the current quark mass was chosen as $10 \mathrm{MeV}$. The particle number at larger momenta depends sensitively on the choice of $\tau$. However, for $\tau \leq 0.15 \mathrm{fm} / \mathrm{c}$ (and thus $\Delta t \lesssim 0.2 \mathrm{fm} / \mathrm{c}$ ) only the high momentum yield is affected. We note that the spectrum of produced quark pairs resembles a thermal distribution of a hot quark-antiquark plasma with a temperature between 200 and $300 \mathrm{MeV}$.

As a further consequence the fermionic degrees of freedom are not necessarily on mass shell during the transition, so that the concept of on-shell quasiparticles and kinetic reactions becomes temporarily invalid. As an example for a particularly interesting nonequilibrium phenomena a single (on mass-shell) gluon which already might be present could in principal decay into a quark-antiquark pair. Such a process is kinematically forbidden if the vacuum does not change its structure. The field theoretical formulation is most conveniently formed in terms of real-time Green functions as appropriate for the study of non-equilibrium processes. The first order decay is contained in the lowest order self-energy insertion $\Sigma \sim G_{0} \cdot D_{0}$. The change in the quark occupation number induced by the (perturbative) interaction of the gluons with the fermionic vacua can be put in the form

$$
\begin{aligned}
& \Delta N_{\mathbf{p}}^{(O u t)}=2 \cdot \lim _{t_{1}=t_{2} \rightarrow \infty} \int d^{3} x_{1} d^{3} x_{2} \int d^{4} x_{3} d^{4} x_{4} \Re\left\{(-i) \varphi_{\mathbf{p} \uparrow}^{(b) \dagger}(1) G_{0}^{\text {ret }}(1,3) \theta\left(t_{3}-t_{4}\right)\right. \\
& \left.\left[\Sigma^{>}(3,4) G_{0}^{<}(4,2)-\Sigma^{<}(3,4) G_{0}^{>}(4,2)\right]\left(\gamma_{0} \varphi_{\mathrm{p} \uparrow}^{(b)}(2)\right)\right\},
\end{aligned}
$$

which resembles the typical form appearing in kinetic transport theories; the two terms in the inner bracket suggest a 'Gain' and 'Loss' contribution. Besides pair production the above 
adiabatic case

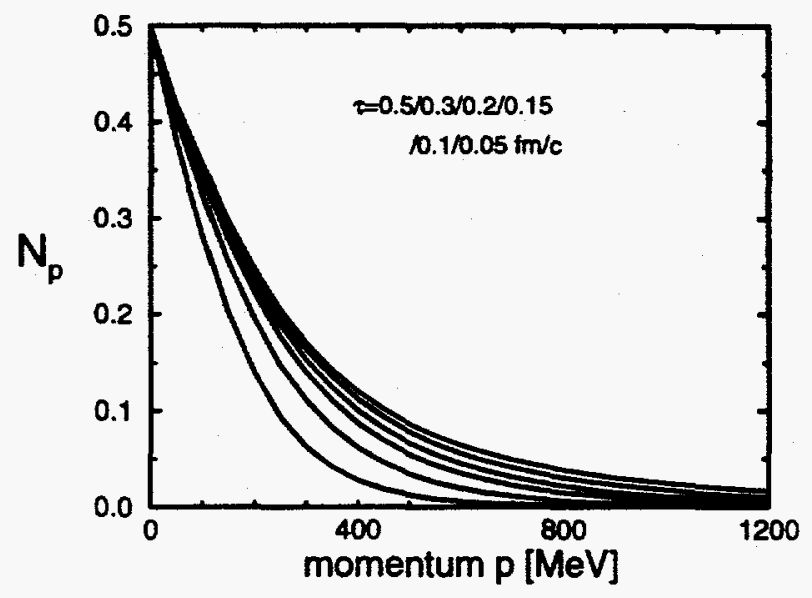

Figure 1: The momentum spectrum of spontanously created quarks for a 'smooth' symmetry restoring transition of the vacuum with a finite duration. The parameter $\tau$ for the transition time is to be read from the left to the right. $m_{\mathrm{c}}=350 \mathrm{MeV}$ and $m_{b} \approx 0 \mathrm{MeV}$.

expression also incorporates the absorption or emission of gluons for the quarks produced in lowest order in the vacuum decay. The cancellation of the infrared singular contributions can be made explicit by spliting the expression into three contributions,

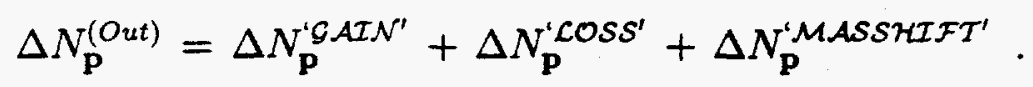

The infrared singularities contained in the 'Gain' term are exactly cancelled by the ones of the 'Loss' part. This gives rise to a new, elegant and 'simple' understanding of the necessary physical origin for the cancellation of the singularities and corresponds to the Bloch-Nordsieck cancellation between real massless photons and virtual photons encountered in the renormalization of the wavefunction in the usual Feynman diagrams [3].

The designation of the 'Masshift' term is based on the fact that, to lowest order in the coupling constant, the (massless) quarks aquire a dynamical mass by the interaction with the gluons:

$$
\Delta N_{\mathbf{p}}^{\prime \mathcal{M} A S S H I F T^{\prime}} \sim\left(\frac{\Delta m_{b}}{E_{\mathbf{p}}^{(b)}}-\frac{\Delta m_{c}}{E_{\mathbf{p}}^{(c)}}\right)
$$

The notion of 'masses' should not be taken literally, because it turns out that they are indeed momentum dependent. In addition, in a thermal environment of gluons it turns out numerically that $\Delta m \sim T^{2}$ and not proportional to $T$, as might be expected from the well known field theoretical calculation of the generation of a dynamical mass at equilibrium. The clear meaning of the present 'mass' generation needs still further investigation [4].

In Figure 2 the fraction $r$ of a gluon 'decaying' into a quark-antiquark pair is depicted. The fraction has the meaning that a specific gluon with a given momentum initiates the production (or annihilation) of $r$ quark pairs during the transition. At low momenta this fraction becomes negative and large; obviously, the low momentum behaviour is unphysical and shows that perturbation theory is not applicable here. It turns out, however, that the 
gluons with higher momenta are mostly decaying into quark pairs. This suggests that such a phenomenon should be of importance in the early stages of the gluon evolution when chiral symmetry restoration takes place.

In future heavy ion experiments at collider energies the initial energy densities are extremely large so that the average momentum of the gluon is high and temperatures of around $500 \mathrm{MeV}$ are expected at the onset of equilibration. Assuming an effective QCD coupling constant $\alpha_{s} \approx 0.4$, a saturation of nearly 50 percent in the quark degrees of freedom is found. Such new nonequilibrium phenomena could give rise to a nonperturbative dynamical creation of quarks and antiquarks (as well as entropy) in the very early stages of the heavy ion reaction. It is tempting to speculate that the total number of produced quarks during such a transition and during the further evolution suffices so that not only the gluonic degrees of freedom become saturated, but also the fermionic ones, before hadronization takes place: The 'hot glue' scenario [5] could turn out to be a hot 'quark gluon plasma' after all.

\section{gluon decay}

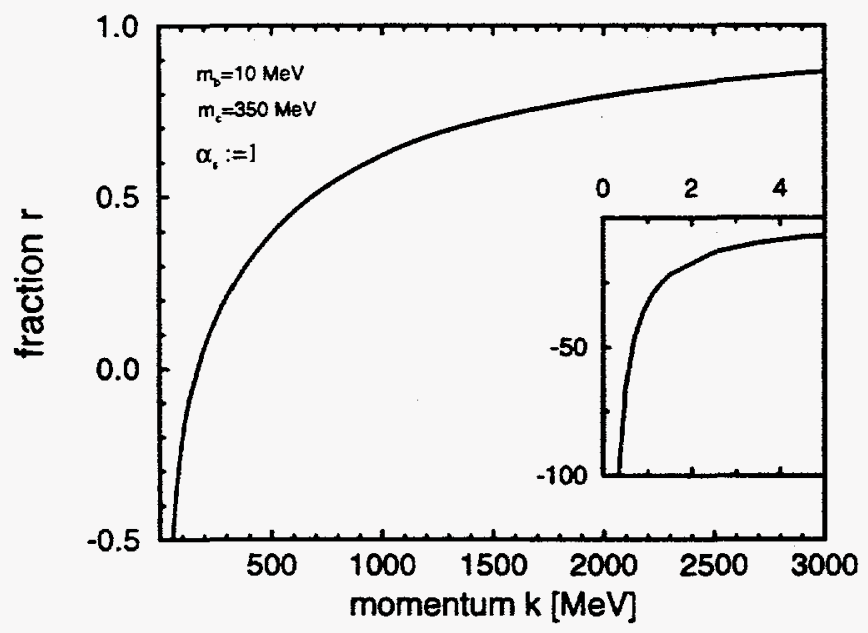

Figure 2: The total fraction of quarkantiquark pairs produced due to the presence of a gluon with a given momenta p. $m_{c}=350 \mathrm{MeV}, m_{b} \approx 0 \mathrm{MeV}$ and $\alpha_{s}$ is set as 1 .

1. C. Greiner was partially supported by the Alexander von Humboldt Stiftung.

2. C. Greiner, Z. Phys. A (in press).

3. H. A. Weldon, Phys. Rev. D26 (1982) 2789.

4. C. Greiner, publication in preparation.

5. E. V. Shuryak, Phys. Rev. Lett. 68 (1992) 3270.

\subsection{Distillation of Strangelets for Low Initial $\mu / T$}

\section{Greiner [1]}

Strangelets may serve as unambiguous signatures for the creation of a quark gluon plasma in heavy ion collisions [2]. The detection of strangelets would verify exciting theoretical 
ideas with consequences for our knowledge of the early universe, the dark matter issue, the dynamics of supernova explosions, neutron stars and the underlying theory of strong interactions [3]. A promising way of producing strangelets out of plasma droplets is through the strangeness separation mechanism which works for droplets with a net amount of baryon number [2]. It can be viewed as being due to the associated production of kaons in the hadronic surrounding with the $s$ quark remaining in the droplet. Earlier studies addressed the production out of a baryon-rich quark-gluon plasma which might be the case for the present $\mathrm{Pb}$ on $\mathrm{Pb}$-experiments at CERN-SPS. In this work [4] we speculate on possible production in the situation of low initial baryon densities and high specific entropies, to match the expected conditions of heavy ion collisions at RHIC (Brookhaven) and LHC (CERN), where the search for strangeness enters in the objective of the ALICE experiment.

The dynamical model employed [5] allows for some important nonequilibrium aspects of the hadronization like pre-freeze-out emission of hadrons, which exhibit important consequences for the further evolution of the droplet, in particular in respect to the net strangeness enrichment, the net baryon concentration and the overall cooling. The initial values for the small (net) anti-baryon number are taken in accordance with the (Poissonian) statistics to be expected due to the fluctuation around their average number of zero (which might indeed also be positive, but small, for the upcoming RHIC facility). Statistically, there are fluctuations at any particular rapidity region where thus a small but net excess in anti-baryon number as well as in net strangeness number might show up. It is worthwhile to point out some analogy with the scenario proposed by Witten [6]: The tiny net baryon excess in the early universe hiding in the 'high-temperature' phase is tremendously small, even compared to the numbers expected at the LHC energies due to fluctuations. The distillation of strange quark matter Witten visualizes as a shrinking, leaking 'balloon', where only neutrinos are allowed to escape, leaving the net baryon density back and trapped inside the balloon. In the present case the mesons take the part of the neutrinos and the question is how efficient their emission is compared to the evaporation of baryons.

We have studied the time evolution of the baryon number inside a droplet for different, yet high initial entropies. It seems that the variation of the intrinsic entropy has no impact on the stability and final baryon number of the strangelet, but it determines the relevant time scale of the hadronization process. This scenario requires a moderate bag constant $B^{1 / 4}<180$ $\mathrm{MeV}$ for the final strangelet to exist as a metastable object. The qualitative reason for the baryon concentration lies in the fact that the baryon density in the hadron phase is typically suppressed by a Boltzmann factor, $\exp \left(-m_{\text {baryon }} / T_{c}\right)$, while in the deconfined phase the density is carried by quarks. Within the present model the distillation mechanism can thus also lead to the formation of very small strangelets of a size $A_{B} \leq 5$ (or (!) anti-strangelets) in the future collider experiments. As we have demonstrated, initial conditions like $|\mu| / T \ll 1$ do not necessarily forbid the condensation of small strange exotic clusters.

1. C. Greiner was partially supported by the Alexander von Humboldt Stiftung.

2. C. Greiner, P. Koch and H. Stöcker, Phys. Rev. Lett. 58, 1825 (1987); C. Greiner, D.-H. Rischke, H. Stöcker and P. Koch, Phys. Rev. D38, 2797 (1988).

3. Proc. Int. Workshop on Strange Quark Matter in Physics and Astrophysics, Nucl. Phys. (Proc. 
Suppl.) 24B.

4. C. Spieles, C. Greiner, H. Stöcker and J.P. Coffin, publication in preparation.

5. C. Greiner and H. Stöcker, Phys. Rev. D44, 3517 (1991).

6. E. Witten, Phys. Rev. D30, 272 (1984).

\subsection{DCC and Strong Electromagnetic Fields}

\section{H. Minakata, B. Müller[1]}

There has recently been considerable interest in the possibility of the formation of a "chirally misaligned" domain of space-time as a result of high energy collisions among protons or heavy nuclei [2-6]. These domains have been called "disoriented chiral condensates" or DCC, because they can be formally described as localized, coherent excitations of the pion fields corresponding to a local rotation of the chiral order parameter of the QCD vacuum. Numerical simulations [3-5] have shown that such a state can spontaneously be created under circumstances where the chiral symmetry of the QCD vacuum has been temporarily restored and then gets broken again during the expansion and cooling process.

Because heavy energetic nuclei are sources of strong electric and magnetic fields, it is of interest to ask whether such fields can have an effect on the formation of DCC's. Some such effects are to be expected because electromagnetic fields break isospin symmetry, which is a subgroup of the $S U(2)_{L} \times S U(2)_{R}$ group of chiral isospin transformations. Expressed differently, electromagnetic fields affect the charged components of the order parameter $\vec{\phi}=$ $(\sigma, \vec{\pi})$ of the QCD vacuum.

As a first step, it is useful to calculate the influence of an external, static, and uniform

electromagnetic field in the framework of the sigma model. Assuming that $\vec{E} \cdot \vec{H}=0$, the result of a one-loop calculation for the effective potential can be represented as:

$$
V_{\text {eff }}=\frac{\lambda}{4}\left(|\phi|^{2}-f_{\pi}^{2}\right)^{2}-f_{\pi} m_{\pi}^{2} \sigma-\mathcal{L}_{\text {em }}^{\prime}\left(F, \sigma^{2}+\pi_{3}^{2}\right),
$$

where $\mathcal{L}_{\mathrm{em}}^{\prime}$ summarizes the one-loop effects and $F=\sqrt{E^{2}-H^{2}}$ ). It is easily seen from this expression that such electromagnetic fields do not influence the orientation of the condensate $\langle\sigma\rangle$ in the ground state, because they leave an $S O(2)$ subgroup $\left(\sigma, \pi_{3}\right)$ of the full $S O(4)$ symmetry of the sigma model invariant. This is not an accident, but simply reflects the fact that the $\pi_{3}$ direction describes a neutral degree of freedom associated with the $\pi^{0}$ field.

This discussion has disregarded a fundamental property of the interaction of electromagnetic fields with composite pseudoscalar fields, namely, the existence of the chiral $U(1)$ (Adler-Bell-Jackiw) anomaly. For electric and magnetic fields that have a parallel component, this gives rise to an additional contribution to the effective potential.

$$
V_{\mathrm{eff}}=f\left(\sigma^{2}+\pi_{3}^{2}\right)-f_{\pi} m_{\pi}^{2} \sigma-\frac{\alpha}{\pi f_{\pi}} \vec{E} \cdot \vec{H} \pi_{3},
$$


clearly breaking the remaining $S O(2)$ symmetry. The minimum in the $\pi_{3}$ direction now occurs at the position

$$
\frac{\pi_{3}}{\sigma}=\frac{\alpha}{\pi f_{\pi}^{2} m_{\pi}^{2}} \vec{E} \cdot \vec{H}
$$

reflecting the relative strengths of the nonconservation of the axial $U(1)$ and the axial $S U(2)$ currents.

The anomaly term only contributes when parallel electric and magnetic fields are present. Parallel field components do appear in collisions of heavy nuclei except in the extreme case of exactly head-on collisions. In order to get an understanding of the orders of magnitude involved, we estimate the electric and magnetic fields generated by fast moving nuclei from the fields at their surface. In the center-of-mass system we have $E \approx H \approx \frac{Z \text { ey }}{4 \pi R^{2}}$, where $Z$ and $R$ are the nuclear charge and radius, respectively, and $\gamma$ is the Lorentz factor in the c. m. system. When the nuclei are ultrarelativistic $(E / A \gg 10 \mathrm{GeV} / \mathrm{u})$, they are Lorentz contracted to pancakes far narrower than $m_{\pi}^{-1}$ and the electromagnetic anomaly provides a quasi-instantaneous "kick" of the ground state away from the $\sigma$-axis. During the brief moment of overlap of the nuclei, the effective potential in the $\pi_{3}$ variable in the vicinity of the normal ground state has the form

$$
V_{\text {eff }}\left(\pi_{3}\right)=\frac{1}{2} m_{\pi}^{2} \pi_{3}^{2}-\left(\frac{Z \alpha \gamma}{2 \pi f_{\pi} R^{2}}\right)^{2} f_{\pi} \pi_{3}
$$

The linear term driving the $\pi_{3}$ field to nonzero values lasts only for a time of order $R / \gamma$, imparting a kick to the conjugate field momentum of order $\Delta \dot{\pi}_{3}=m_{\pi}^{2} / 60$ for two colliding $\mathrm{Au}$ nuclei at RHIC $(\gamma=100)$. This results in a coherent oscillation of the chiral order parameter in the $\pi_{3}$ direction over a volume of nuclear dimensions. Although the amplitude is quite small on the scale of $m_{\pi}$, its coherence over a region much larger than $m_{\pi}^{-1}$ could be important because it establishes an explicit breaking of isospin symmetry in the initial conditions for the formation of a DCC state. It would be interesting to explore these consequences further in a dynamical model of DCC formation.

1. This research was supported in part by the National Science Foundation. B. M. gratefully acknowledges the hospitality of the Department of Physics of Tokyo Metropolitan University during his visit in July 1994.

2. J. D. Bjorken, Acta Phys. Pol. B23, 561 (1992).

3. K. Rajagopal and F. Wilczek, Nucl. Phys. B404, 577 (1993).

4. S. Gavịn, A. Gocksch, and R. D. Pisarski, Phys. Rev. Lett. 72, 2143 (1994).

5. M. Asakawa, Z. Huang, and X.-N. Wang, Phys. Rev. Lett. 74, 3126 (1995).

6. K. Rajagopal, The chiral phase transition in QCD: Critical phenomena and long wavelength pion oscillations, preprint, Harvard University (April 1995). 


\title{
1.6 Lyapunov Exponent and Plasmon Damping Rate
}

\author{
T. S. Biró, C. Gong, B. Müller
}

Numerical studies of Hamiltonian $\mathrm{SU}(N)$ lattice gauge theory in $(3+1)$ dimensions have shown that the gauge fields exhibit chaotic behavior in the classical limit [1,2]. The numerical value of the largest positive Lyapunov exponent $\lambda_{0}$ has been obtained for $S U(2)$ and $S U(3)$ with the result

$$
\lambda_{0}=\frac{2}{3} c_{N}\left(N^{2}-1\right) g^{2} T \approx \begin{cases}0.34 g^{2} T & (N=2), \\ 0.53 g^{2} T & (N=3) .\end{cases}
$$

These values for $\lambda_{0}$ coincide, apart from a factor of two, with those of the damping rate of a thermal plasmon at rest, obtained in the framework of thermal perturbation theory [3]:

$$
\gamma_{0} \approx 6.635 \frac{N}{24 \pi} g^{2} T= \begin{cases}0.176 g^{2} T & (N=2), \\ 0.264 g^{2} T & (N=3) .\end{cases}
$$

Here we attempt to justify this connection and to explain the origin of the factor $\lambda_{0} / \gamma_{0}=2$.

We reviewed the numerical "measurement" of the Lyapunov exponent in classical lattice gauge theory. The exponential growth rate of a small perturbation in the magnetic energy density used in those calculations is equal to twice the growth rate of fluctuations in the elementary field variable, in the continuum limit the vector potential. This explains the factor of two between $\lambda_{0}$ and $\gamma_{0}$. We then demonstrated that in classical calculations the linear perturbation propagation corresponding to the equations of motion of a chaotic dynamical system has in general a Fourier spectrum of imaginary frequencies. The Lyapunov exponent is equal to the magnitude of those imaginary frequencies.

We argue that the chaotic dynamics of the classical system act like a thermal ensemble averaging the linearized equation over stochastic frequencies. The square of these frequencies can either be positive or negative. In this case the damping rate and the plasma frequency of the classical elementary field fluctuations are related to the mean value and the width of the probability distribution of frequency squares. The final result of these considerations is that the Lyapunov exponent as defined in [1] measures twice the damping rate of classical gauge field fluctuations on the lattice. We finally show that the quantum field theoretical calculation of the thermal damping rate at rest in hot perturbation theory in the leading $\mathcal{O}\left(g^{2} T\right)$ order survives in the classical limit $(\hbar \rightarrow 0)$.

We note that some elements of the argument are heuristic, in particular, the replacement of the long-time average of the growth rate of fluctuations around a specific field configuration by the thermal average. This reasoning assumes that the growth rate, or equivalently the plasmon damping rate, depends only on coarse-grained properties of the gauge field. We believe that this is so, because the one-loop calculation of the damping rate $\gamma_{0}$ only involves soft loop momenta and hence does not depend on details of the short-distance fluctuations of the gauge field.

1. B. Müller and A. Trayanov, Phys. Rev. Lett. 68, 3387 (1992).

2. C. Gong, Phys. Lett. B298, 257 (1993). 
3. E. Braaten and R. D. Pisarski, Phys. Rev. D42, 2156 (1990).

\title{
1.7 Dynamics of Soft Modes in Thermal Gauge Theories
}

\author{
T. S. Biró, S. G. Matinyan, B. Müller
}

The numerical results $[1,2]$ showing chaoticity of the thermalized $\mathrm{SU}\left(N_{c}\right)$ Yang-Mills field (in the microcanonical approach) appear to be at odds with the observation [3] that the thermal mass term, $m_{\mathrm{PL}}^{2}=\frac{1}{9} N_{c} g^{2} T^{2}$, destroys the chaotic behavior of small amplitude collective field oscillations (plasmons). In order to pursue this issue, we derived an effective equation of motion for the plasmon field, which takes into account the color-changing nonabelian interactions between plasmons and hard thermal gluons. We find that these interactions can be accounted for by Feynman rules for the plasmon field and its higher order correlations derived from the Yang-Mills Lagrangian, which generate stochastic mass and source terms for the collective plasma waves.

The equation of motion for the plasmon field contains, besides the classical contributions stemming from the first variation of the Yang-Mills action, two further contributions describing interactions with hard thermal gluons. One is the self energy term $m_{\mathrm{PL}}^{2}$, which arises from the dressed plasmon two-point function by detaching a plasmon field factor. This accounts for the real and imaginary part of the hard thermal loop self energy. Also a stochastic source term, $q_{\mathrm{PL}}$, stemming from the plasmon one-point function, occurs. This equation can be written in the form [4]

$$
\left(D+m_{\mathrm{PL}}^{2}+\sum_{n \geq 3} \Gamma_{n} A^{n-2}\right) A=q_{\mathrm{PL}}
$$

Here $D$ is the differential operator corresponding to the classical equation of motion which includes the plasmon field $A$ in nonlinear combinations. $\Gamma_{n}$ is the hard thermal loop contribution to the soft $n$-point function, while $m_{\mathrm{PL}}^{2}=\Gamma_{2}$ and $q_{\mathrm{PL}}=-\Gamma_{1}$ are the hard thermal loop contributions to the 2- and 1-point functions.

In the solution $\langle A\rangle=\left\langle\left(D+m_{\mathrm{PL}}^{2}\right)^{-1} q_{\mathrm{PL}}\right\rangle+\left\langle A_{\text {hom }}\right\rangle$, the stochastic source term $q_{\mathrm{PL}}$ is of the adjoint representation of the gauge group and hence has vanishing thermal average, but it can combine with color non-singlet terms in the stochastic part of the self-energy operator $m_{\mathrm{PL}}^{2}$ to contribute to the slow plasmon field $A$. This part describes a color exchange interaction between the slow plasmon and the fast oscillating hard thermal gluons.

The eigenvalues and eigenvectors of the operator $\left(D+m_{\mathrm{PL}}^{2}\right)$ are sensitive not only to the color singlet part of the plasmon self-energy, but also to the stochastic non-singlet part which corresponds to an exchange of color quantum numbers between the plasma wave and the medium. We have shown in a simple model, derived as a special case of the full YangMills field dynamics, how the stochastic off-diagonal components of the mass operator can restore chaoticity to the mean field dynamics. Note that the non-singlet components of $m_{\mathrm{PL}}^{2}$ and $q_{\mathrm{PL}}$ are closely related to the color transport properties of the nonabelian plasma. The 
color conductivity coefficient $[5,6]$ of thermal Yang-Mills fields is of order $\mathcal{O}\left(g^{2} T\right)$, and hence we expect that the chaotic dynamics of the plasma modes will also appear at this order in perturbation theory.

1. S.G. Matinyan, G.K.Savvidy and N.G. Ter-Arutyunyan-Savvidy, JETP 53, 421 (1981).

2. B. Müller and A. Trayanov, Phys. Rev. Lett. 68, 3387 (1992).

3. J.-P. Blaziot and E. Iancu, Phys. Rev. Lett. 72, 3317 (1994).

4. T. S. Biró, S. G. Matinyan, and B. Müller, preprint DUKE-TH-95-90, submitted to Phys. Rev. Lett.

5. A.V. Selikhov and M. Gyulassy, Phys. Lett. B316, 373 (1993).

6. H. Heiselberg, Phys. Rev. Lett. 72, 3013 (1994).

\title{
1.8 Collisions of Yang-Mills Wavepackets
}

\author{
C.-R. Hu, S. G. Matinyan, B. Müller, A. Trayanov
}
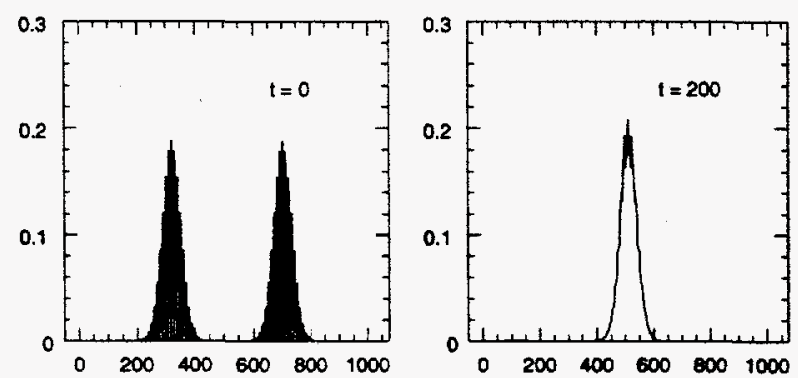

Figure 1: Space-time evaluation of two
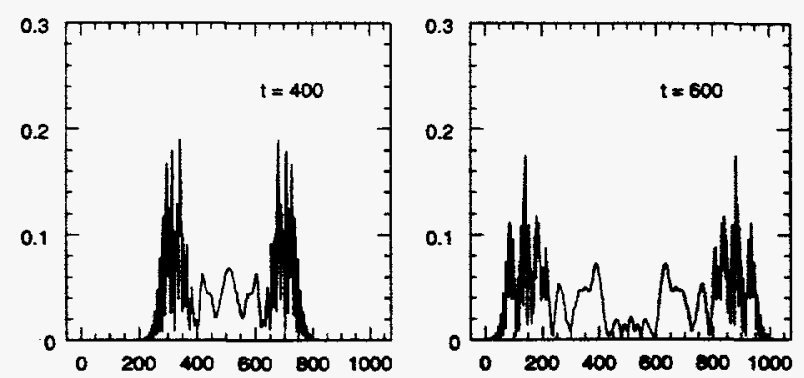
wavepackets with orthogonal initial polarizations.

We have developed a nonperturbative approach to multiparticle production in strong (QCD) as well in electroweak scattering processes based on the classical analogue to high-energy collisions [1]. From this point of view the problem is connected to the cardinal question: does there exist energy transfer from fast (high frequency) classical wave modes corresponding to two high energy particles to slow (low frequency) modes describing multiparticle final states?

In our previous study [2] we have shown, on the basis of a simple example, that this behavior has its roots in the nonintegrable character of non-abelian gauge theories. In the 
present paper we have developed a more realistic approach based on the study of the collisions of two $\mathrm{SU}(2)$ wavepackets on a one-dimensional lattice. We investigated the collision of wavepackets as a function of amplitude and momentum distribution. We have found that essentially non-abelian initial configurations of the colliding wavepackets result in final configurations (see Figure 1) with dramatically different momentum distributions. An initially abelian configuration does not change after the collision. These results support the approach based on the existence of new types of classical trajectories with multiparticle boundary conditions [3]. We explain their relevance to the studies of scattering amplitudes in the semiclassical approximation.

1. C. Hu, S. G. Matinyan, B. Müller, A. Trayanov, T. M. Gould, S. D. Hsu, and E. Poppitz, Wavepacket Dynamics in Yang-Mills Theory, submitted to Phys. Rev. D..

2. C. Gong, S. G. Matinyan, B. Müller and A. Trayanov, Phys. Rev. D49, R607 (1994).

3. T. M. Gould, S. D. M. Hsu, and E. R. Poppitz, Nucl. Phys. 347, 83 (1995).

\subsection{Interface Tension and Entropy}

\section{B. Müller}

The dynamics of phase transitions in the early universe and in relativistic heavy ion collisions has recently attracted a great deal of interest. When the transition is of first order, it proceeds by formation of critical droplets of the new phase which grow into larger bubbles until the transformation of the high-temperature phase is completed. The high-temperature phase transition of quantum chromodynamics (QCD) is believed to be of first order in the case of three light quark flavors. When the influence of dynamical quarks on the thermodynamical variables is neglected, the transition is also of first order, with a latent heat $\Delta \varepsilon \approx 2 T_{c}^{4}$, where $T_{c}$ is the critical temperature. Numerical results for the interface tension between the confining and deconfining phases of QCD have been obtained by computer simulations of the lattice $\mathrm{SU}(3)$ gauge theory [1]. These studies have shown surprisingly small values of the interface tension: $\sigma / T_{c}^{3} \approx 0.02-0.03$. Since the free energy of the critical droplet is proportional to $\sigma^{3}$, the small value of $\sigma$ makes significant supercooling in the cosmological context very unlikely.

The reason for the smallness of $\sigma$ in the QCD phase transition is not well understood. Moreover, it is not quite clear whether the value, which is obtained under the condition that both phases are in thermal equilibrium, is the relevant quantity in more dynamical scenarios where the transition proceeds as a nonequilibrium process. The interface tension obtained in lattice calculations is defined as the energy of the interface per unit area at constant temperature, and should be properly called the isothermal interface tension $\sigma_{T}$. One could, in principle, also define the interface tension at constant entropy, or adiabatic interface tension $\sigma_{A}$, which would be relevant in entropy conserving processes. In order to relate $\sigma_{A}$ and $\sigma_{T}$ it is necessary to calculate the entropy associated with the interface between the two phases. 
We have explored these issues by studying a very simple, analytically tractable model of an interface between two regions of space with different values of the order parameter in a relativistic quantum field theory. This model, a scalar field theory with two degenerate vacua, is defined by the Lagrangian

$$
\mathcal{L}=\frac{1}{2} \partial_{\mu} \phi \partial^{\mu} \phi-\frac{\lambda}{4}\left(\phi^{2}-\phi_{0}^{2}\right)^{2} .
$$

The degenerate vacuum states are $\langle\phi\rangle= \pm \phi_{0}$. Perturbative excitations in both phases have the mass $m=\phi_{0} \sqrt{2 \lambda}$. We find for the interface entropy [2]

$$
S_{A}=-\frac{\partial F}{\partial T}=A T^{2}\left(3 f(\beta m)-\beta m f^{\prime}(\beta m)\right),
$$

where $A$ is the area of the interface and

$$
\begin{aligned}
f(\beta m) & =\frac{1}{2 \pi} \sum_{n=1}^{\infty} \frac{1}{n^{3}}\left[1+\left(n \beta m \frac{\sqrt{3}}{2}+1\right) \exp \left(-n \beta m \frac{\sqrt{3}}{2}\right)\right. \\
& \left.-\frac{1}{2 \pi} \int_{-\infty}^{\infty} d k \rho(k)\left(n \beta \sqrt{k^{2}+m^{2}}+1\right) \exp \left(-n \beta \sqrt{k^{2}+m^{2}}\right)\right] .
\end{aligned}
$$

We find that the (isothermal) surface tension of the interface between two phases will generally be lowered by the surface entropy from what one might expect on purely energetic grounds. If the interface is thin-walled, compared with the wavelength of thermal fluctuations, the entropy is approximately given by that of a thermally vibrating relativistic membrane, growing like $T^{2}$. If the interface is thick, the entropy grows only linearly with the temperature, but this suffices to render the surface tension negative above some temperature. Of course, the interface will only be stable in a narrow range of temperatures where metastable phase coexistence is possible. However, this argument makes it clear why potential energy and entropy terms can almost cancel each other in a theory like QCD where all constants are controlled by a single parameter, $\Lambda_{\mathrm{QCD}}$. The adiabatic surface tension $\sigma_{A}$ does not exhibit such a cancellation and always has a value exceeding the pure potential energy density of the domain wall.

1. E.g.: Y. Iwasaki, K. Kamaya, L. Kärkkäinen, K. Rummukainen, and T. Yoshie, Phys. Rev. D49, 3540 (1994).

2. B. Müller, DUKE-TH-94-75.

\section{Effective Theories for Hadrons and Nuclei}

\subsection{Kaon-Nucleon-Nucleon Couplings for Decays of Hypernuclei}

\section{R. Springer}

The power of Heavy Baryon Chiral Perturbation Theory $(H B \chi P T)[1]$ is that it includes baryons in the description of nonperturbative QCD that chiral Lagrangians provide. The 
resulting effective theory can be used to predict observables measured at sufficiently low energies so that the expansion in momentum transfer over chiral symmetry breaking scale $\left(\Lambda_{\chi} \sim 1 \mathrm{GeV}\right)$ converges. $H B \chi P T$ has been used to investigate the nonleptonic decays of hyperons into nucleons and pions [2]. The analysis covered both s-wave and p-wave processes, and the disturbing discovery was made that the parameters of the theory which experimental s-wave properties would dictate were not compatible with p-wave processes and vice-versa. At that point, there was speculation that perhaps the chiral Lagrangian was not valid for describing nonleptonic hyperon decays [3], which brought into question its validity for other observables as well. We use $H B \chi P T$ to perform a calculation for the KNN coupling, both $\mathrm{s}$-wave and $\mathrm{p}$-wave processes, to one loop order, to investigate whether the results found in Ref.[2] were an anomalous accident, or an indication of a trend that might invalidate the use of chiral Lagrangians in some cases.

The weak decays of hypernuclei provide information on the KNN coupling. The $\Lambda$ particle in the hypernucleon is Pauli blocked (in nuclei with $A \sim 12$ ) from decaying into a nucleon and a pion, as would happen in free space. Instead, the dominant mode of relaxation for a hypernucleus takes place via the scattering of the $\Lambda$ off another nucleon through meson exchange, with the emission of two nucleons in the final state (each with momentum large enough to be above the Fermi sea for the nucleus). The meson exchanged may be a pion (and this case has been extensively studied in models) but the failure to explain the measured ratio of neutron to proton induced decay rates points to the importance of short distance physics like the exchange of kaons. This is where the weak KNN coupling appears. There are three possible vertices: $\bar{p} p K^{0}, \bar{p} n K^{+}$, and $\bar{n} n K^{0}$. We find [4] that, unlike the hyperon case where the p-wave vertices suffered from corrections at the $100 \%$ level when $\mathrm{SU}(3)$ breaking effects were included, both the $\bar{p} n K^{+}$, and $\bar{n} n K^{0}$ are well behaved, and display the expected $30 \%$ corrections. The $\bar{p} p K^{0}$ vertex, on the other hand, does suffer from very large corrections. This once again seems to be the result of an anomalously small tree level p-wave amplitude for the $\bar{p} p K^{0}$ coupling, which is what happened for the $\mathrm{p}$-wave hyperon decays. These results lend credence to the idea [2] that the chiral Lagrangian technique itself is not flawed, but simply that substantial corrections will appear when the lowest order terms are unexpectedly small. Further, our calculation of the KNN couplings can be incorporated into hypernuclei decay codes to see if they are important for finally understanding. some of the hypernuclei measurements such as the neutron to proton induced decay ratio.

1. E. Jenkins and A. Manohar, Proceedings of the workshop on Effective Field Theories of the Standard Model, ed. U. Meissner, World Scientific (1992).

2. E. Jenkins, Nucl. Phys. B375, 561 (1992).

3. C. Carone and H. Georgi, Nucl. Phys. B375, 243 (1992).

4. M. Savage and R. Springer, DUKE-TH-94-77. 


\subsection{Limits on Possible T-Violating, P-Conserving Observables}

\section{R. Springer}

Nuclear systems provide a very interesting place in which to search for the violation of fundamental symmetries. Polarization techniques and well-understood beam and detector technology allow for the possibility of extremely sensitive measurements of Standard Model symmetries. Much interest of late has come from a community which investigates time reversal $(T)$ violating, but parity reversal $(P)$ conserving observables [1]. This is not the same process which has been measured in the neutral kaon system, where interactions portray a T-violating, but also P-violating behavior. Symmetry violation in the neutral kaon system is explainable (though not necessarily encompassed) by the Standard Model of Weak Interactions plus QCD. The forum of T-violating, P-conserving (TVPC) observables discussed here, however, lies outside the realm of Standard Model physics. Current experiments use polarized neutron beams on heavy nuclei to yield limits on TVPC processes at the $10^{-3} g$ level [1], where $g$ is the strong coupling constant at nuclear energies.

To show how unknown physics at a high energy scale might give rise to TVPC effects, we made arguments based on general properties of effective theories and power counting methods [2] to demonstrate the relevance of allowed terms in an effective Lagrangian. The power of these arguments is that we need not know the exact form of the unknown physics at high energies to know how they will affect measurements at nuclear energies. This concept is invoked to explain the success of all theories from Newton's Laws to the Standard Model of Weak Interactions. Though neither may be the most fundamental theory dictating the physics we see, each is quite valid and useful in an appropriately specified regime of interaction. As sensitivity to higher energy processes and heavier mass particles increases, we may begin to see deviations from the predictions of our effective theories (of Newton or of Glashow, Weinberg, and Salam) which signal the presence of new physics. It is at this level that we can explore the terrain of physics that may give rise to TVPC operators in an effective Lagrangian. This is an exercise in building operators which have the desired TVPC property, and are of the lowest dimension possible. (The lowest order symmetry conserving QCD terms are of dimension four.) New physics will generate (nonrenormalizable) higher order effective operators at nuclear energy scales. As the dimension of the operator increases beyond four, each operator will be suppressed by the large mass scale where the new physics exists.

$$
\mathcal{L}_{\text {eff }}=\mathcal{L}_{0}+\frac{p}{\Lambda} \mathcal{L}_{1}+\frac{p^{2}}{\Lambda^{2}} \mathcal{L}_{2}+\ldots
$$

where $\mathcal{L}_{0}$ contains the dimension four (Standard Model) operators, $\mathcal{L}_{1}$ contains operators of dimension five, and so on. The typical momentum scale, $p$, would here represent momentum transfers involved in the TVPC experiments mentioned above, and $\Lambda$ would be the size of, for instance, some new massive particle which can mediate TVPC interactions. The lowest dimension operator found with the desired behavior was of dimension seven [3]. For $\Lambda \geq 100$ $\mathrm{GeV}$, which seems reasonable based on searches conducted at high energy accelerators, this implies that the size of TVPC processes will be at about the $10^{-6} \mathrm{~g}$ level. Unless the coefficient 
multiplying the operator in the effective Lagrangian is unexpectedly large (which of course could happen; witness the 20 -fold enhancement that is the " $\Delta I=1 / 2$ rule"), experiments looking for TVPC interactions may need to increase their sensitivity by several orders of magnitude before something interesting happens.

1. For instance, Time Reversal Invariance and Parity Violation in Neutron Reactions, ed. C.R. Gould, D. Bowman, and Y.P. Popov, World Scientific, Singapore, (1994).

2. Howard Georgi, Ann. Rev. Nucl. Part. Sci. 43, 209 (1994).

3. J. Engel, P. Frampton, R. Springer, preprint DUKE-TH-95-93.

\subsection{Baryon Magnetic Moments from a Chiral Extrapolation of Lattice Results.}

\section{R. Springer}

Two of the best tools for investigating nonperturbative QCD in baryons are lattice gauge theory and chiral Lagrangians. They are complementary techniques in that they both look at very different limits of $\mathrm{QCD}$, and are sensitive to different physics. For instance, loop corrected predictions from chiral Lagrangians probe mesonic dressing and give the structure of terms nonanalytic in quark masses, while lattice calculations are more sensitive to short distance physics and involve terms analytic in quark masses. For the lattice, quarks are taken to be heavier than their real masses; for chiral methods, they are taken to be lighter. Our hope is that using information from both methods will minimize the errors made from the assumptions of either, yielding a better understanding of nonperturbative QCD in baryons.

We first looked at the octet baryon magnetic moments because there is experimental data with which to compare our results. The chiral predictions for this system are far from accurate, for reasons that are not particularly clear (but may eventually shed light on the role of strange mesons in this process) [1]. Lattice calculations are also available [2], but extrapolation to predictions involving physical quark masses was done using only terms linear in the quark mass. The chiral calculation gives the possible forms involving nonanalytic corrections. Taken together, one knows the possible terms that will appear, order by order, in an effective Lagrangian,

$$
L_{e f f}=c_{0}+c_{1} m_{q}^{1 / 2}+c_{2} m_{q} \log \frac{m_{q}}{\Lambda_{\chi}}+c_{3} m_{q}+\ldots
$$

where $\Lambda_{\chi} \sim 1 \mathrm{GeV}$ is the chiral symmetry breaking scale, $m_{q}$ is a light quark mass, and the coefficients $c_{i}$ may be estimated from the lattice, from loop corrected chiral Lagrangians and/or fits to lattice data and real data. Higher order terms of course exist, but are suppressed by ever-higher powers of $\Lambda_{\chi}$. The best fits are obtained when $c_{2}$ is taken as a free parameter instead of fixed by the chiral prediction (there are enough data from lattice calculations to make such fits meaningful) [3]. Study of this system is ongoing, with attention paid not only to variations of the lightest meson masses with quark mass, but also with respect to 
kaon mass variation. Because of our extensive study into baryon decuplet properties using chiral Lagrangians, and the existence of lattice data on the same observables, we will next apply these ideas [4] to the decuplet of baryons.

1. E. Jenkins, M. Luke, A. Manohar, M. Savage, Phys. Lett. B302,482 (1993).

2. D. Leinweber, R Woloshyn, T. Draper Phys. Rev. D43, 1659 (1991).

3. M. Butler, D. Leinweber, R. Springer, in preparation

4. an example appears in D. Leinweber, T. Cohen, Phys. Rev. D47, 2147 (1993).

\subsection{Photoproduction of $\pi^{0}$ on Light Nuclei}

\section{S. Beane}

In recent times, there has been increasing interest in applying the method of chiral Lagrangians, or chiral perturbation theory $(\chi \mathrm{PT})$, to processes involving more than a single nucleon. This interest is motivated by the desire to determine what aspects of nuclear physics can be understood on the basis of the chiral symmetry of QCD. We have studied threshold neutral pion photoproduction on light nuclei in the framework of baryon chiral perturbation theory [1], following a method pioneered by Weinberg [2]. The overall strategy is best exhibited diagramatically (see Figure 1). The existence of nuclear bound states demands a modification of the usual rules; $\chi \mathrm{PT}$ is used to obtain the irreducible kernel $\mathrm{I}$, which is then sewn to the nuclear wave functions. In principle, the nuclear wavefunctions are calculable in $\chi \mathrm{PT}$, however this technology is still in its infancy, and so in the deuteron calculations use is made of the phenomenologically successful Bonn wave function [3]. A general formula was obtained for the electric dipole amplitude in the special case of neutral pion photoproduction on a light nucleus. To third order in small momenta, the amplitude is a sum of 2-and 3-body interactions with no undetermined parameters. Unfortunately, the single nucleon sector (2body interactions) is problematic. The electric dipole amplitudes suffer from convergence problems in $\chi \mathrm{PT}$. On the other hand, the experimental situation regarding the proton electric dipole amplitude, $E_{0+}^{\pi^{0} p}$, is still unclear due to a rapid variation of the amplitude near threshold. Currently new measurements of $E_{0+}^{\pi^{0} p}$ are underway at Mainz and Saskatoon [4]. Moreover, the neutron amplitude has not been measured. Evidently the sensible thing to do is to make a best phenomenological estimate of the single scattering contribution. The chiral prediction without the triangle graphs would appear to be a reasonable phenomenological estimate of these contributions. With this phenomenological input from the 2-body sector, the result for neutral pion photoproduction on the deuteron is in agreement with experiment. The total theoretical electric dipole amplitude is given by

$$
E_{d}=(-1.33-2.66) \times 10^{-3} / M_{\pi^{+}}=-3.99 \times 10^{-3} / M_{\pi^{+}},
$$

where the contributions are divided into 2 - and 3-body effects, respectively. The experimental value is [5]: 


\begin{tabular}{||c|c|c|c|c||}
\hline AMPLITUDE & $E_{0+}^{\pi^{0} p}$ & $E_{0+}^{\pi^{0} n}$ & $E_{d}^{s s}$ & $E_{d}$ \\
\hline $\mathcal{O}\left(q^{3}\right)$ incomplete & -2.24 & 0.5 & -1.33 & -3.99 \\
\hline $\mathcal{O}\left(q^{3}\right)$ & 0.96 & 3.7 & 3.6 & 0.94 \\
\hline experiment & $-2.0 \pm 0.2(?)$ & $?$ & - & $-3.74 \pm 0.25$ \\
\hline
\end{tabular}

Table 1: The importance of the single scattering contribution: The $\chi$ PT predictions at $\mathcal{O}\left(q^{3}\right)$ without the triangle graphs $\rightarrow$ serving as a phenomenological estimate- lead to agreement with the experimental value of $E_{d}$.

$$
E_{d}^{\exp }=(-3.74 \pm 0.25) \times 10^{-3} / M_{\pi^{+}} .
$$

Hence the simple picture provided by chiral symmetry does fairly well. In particular, the importance of the 3-body correction (charge-exchange contribution) emerges as a natural consequence of chiral symmetry. However, strictly speaking the $\mathcal{O}\left(q^{3}\right)$ result fairs badly, as is made clear in Table 1. This is, of course, a consequence of the theoretical failure in the single nucleon sector. Moreover, in order to be convinced of the soundness of this result one would have to calculate to $\mathcal{O}\left(q^{4}\right)$; this would involve 3-body loops as well as tree level 4-body effects. Charged photoproduction-which will soon be measured in Saskatoon, and should be free of convergence problems-is currently under investigation.

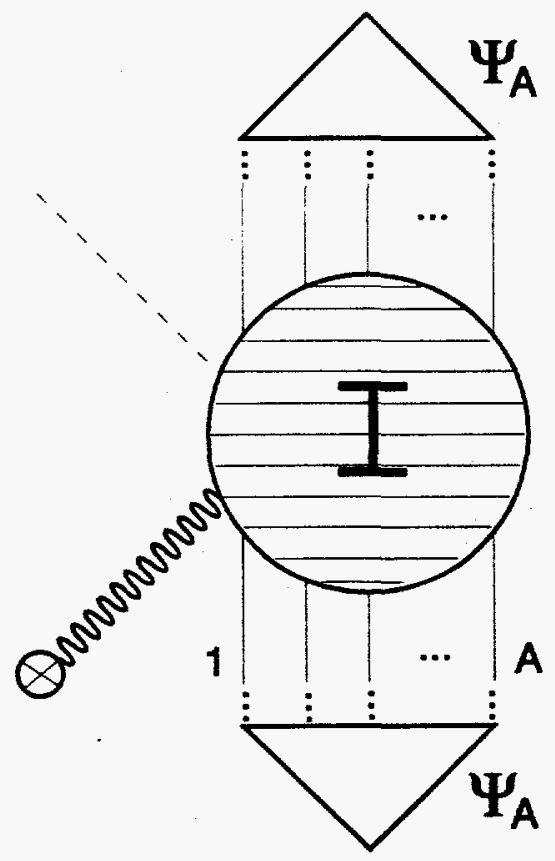

Figure 1: The anatomy of a matrix element. The $\Psi_{A}$ 's correspond to the nuclear wave functions. $\mathcal{I}$ - the sum of all $A$-nucleon irreducible graphs-is obtained in baryon chiral perturbation theory.

1. S.R. Beane, C.Y. Lee, and U. van Kolck, DUKE-TH-95-88.

2. S. Weinberg, Phys.Lett. B295, 114 (1991).

3. R. Machleidt, K. Holinde, and Ch. Elster, Phys.Rep. 149, 1 (1987). 
4. V. Bernard, N. Kaiser, and Ulf-G. Meißner,CRN-95-3, hep-ph/9501384.

5. P. Argan et al, Phys. Rev. Lett. 41, 629 (1978).

\section{Other Topics}

\subsection{Renormalization Group Approach to Field Theory at Finite Temperature}

\section{S.-B. Liao, M. T. Strickland}

In recent years, intensive efforts have been devoted to quantum field theory at finite temperature, a subject with wide applications in areas such as the evolution of the early universe and its cosmological consequences, the deconfinement phenomena and formation of the quark-gluon plasma, and the critical behavior of condensed matter systems near the phase transition. To investigate these issues, one generally utilizes the finite-temperature effective potential approach in the spirit of the perturbative loop expansion. However, in the high temperature limit, perturbation theory becomes unreliable [1] since the presence of infrared (IR) divergences may destroy the correspondence between the expansions of loops and the coupling constant. Certain higher loop contributions such as the "daisy" and "superdaisy" diagrams that contribute to the same order in the coupling constant must also be incorporated [2] for computing the critical transition temperature $T_{c}$ and determining the nature of the phase transition. In gauge theories, it has been shown that the "hot thermal loops" need to be resummed in order to obtain a gauge independent gluon damping rate [3].

We have recently investigated the use of renormalization group flow equations for studying the finite temperature behavior of a scalar $\lambda \phi^{4}$ theory [4]. This formulation not only takes into consideration the dominant higher loop diagrams without complicated analytical order by order resummation, it also characterizes the flow pattern of the theory for arbitrary temperature $T$ as well as infrared-momentum scale $k$. This approach is similar to the series of works by Tetradis and Wetterich [5]. However, instead of using a smooth momentum smearing function which leads to an integro-differential RG equation, a sharp momentum cut-off is used yielding a $\mathrm{RG}$ flow equation which takes on the form of non-linear partial differential equation.

Consider the following scalar Lagrangian:

$$
\mathcal{L}=\frac{1}{2}\left(\partial_{\mu} \phi\right)^{2}+V(\phi)
$$

At finite temperature, the RG improved blocked potential $U_{\beta, k}(\Phi)$ associated with the blocked field $\Phi(x)$ is characterized by the following differential RG equation [1]:

$$
k \frac{\partial U_{\beta, k}}{\partial k}=-\frac{k^{3}}{4 \pi^{2}} \sqrt{k^{2}+U_{\beta, k}^{\prime \prime}}-T \frac{k^{3}}{2 \pi^{2}} \ln \left[1-e^{-\beta \sqrt{k^{2}+U_{\beta, k}^{\prime \prime}}}\right] .
$$



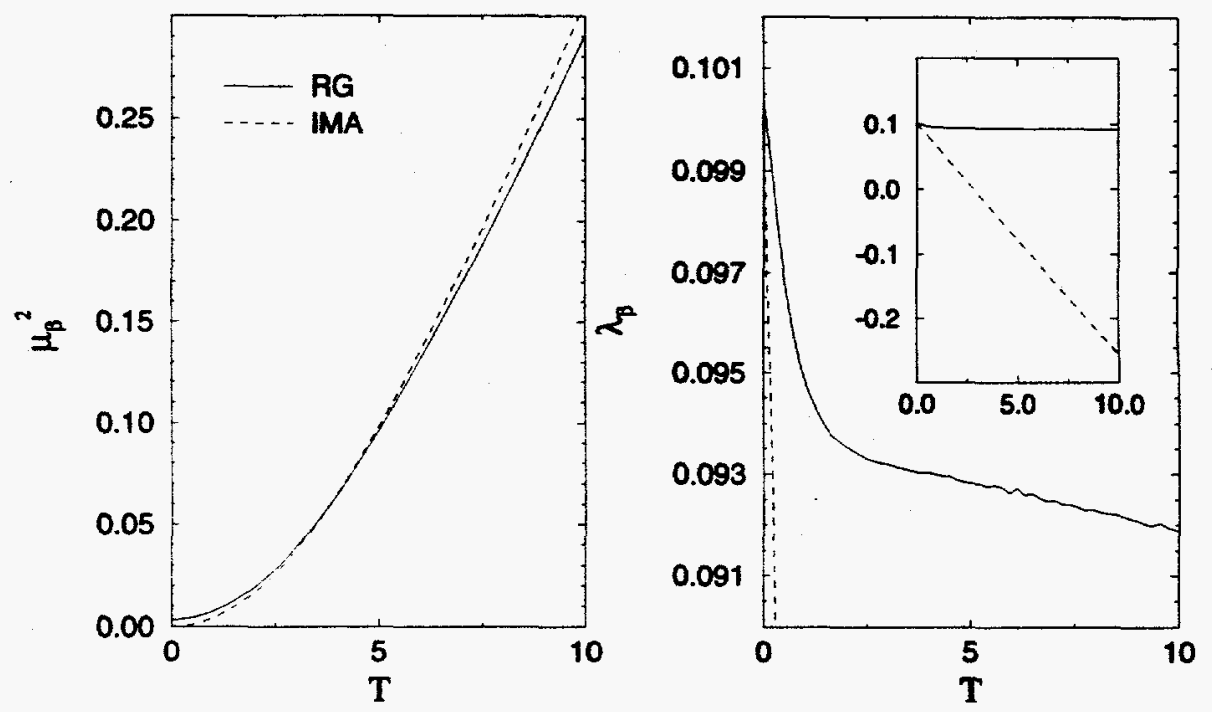

Figure 1: Temperature dependence of the mass $\mu_{\beta}^{2}$ and the coupling constant $\lambda_{\beta}$ at $k=0$.

Equation (2) is obtained by first computing the finite-temperature blocked potential to one-loop order followed by a RG improvement to take into account all possible nonoverlapping daisy, superdaisy and higher-loop diagrams which strongly modify the theory in the high $T$ and small $k$ limits. In this construction of the RG the contributions of modes which have been integrated out are naturally retained for the integration of the next, and therefore the interactions among the modes are properly taken into account. In addition, during the course of mode elimination, irrelevant operators defined with respect to the ultraviolet (UV) fixed point continue to be generated and their effects incorporated as well. To describe the full theory, however, the effects of wavefunction renormalization and higher-order derivative terms on the RG flow must also be investigated.

With this flow equation we have studied the high and low $T$ limits of $\lambda \phi^{4}$ theory. In the low temperature limit it was verified that the $O(4)$ and $O(3)$ blocked flow equations give the same renormalized parameters. In the high temperature limit it was found that only a partial dimensional reduction occurs and that the coupling constant $\lambda_{\beta, k}$ approaches a positive constant value as seen in Figure 1. In the broken phase it was shown the RG approach correctly predicts a vanishing imaginary contribution in $U_{\beta, k}(\Phi)$ after symmetry restoration. This is to be contrasted with the perturbative independent mode approximation for which the imaginary part persists after symmetry restoration.

Various interesting issues can be explored with this RG scheme. For example, one can use this approach to study the nature of the phase transition for the electroweak theory. For $\lambda_{R}<<g_{R}^{2}$, where $g_{R}$ is the coupling constant for the gauge fields, one would expect a first order transition which is required for explaining the asymmetry of the baryogenesis. For the Yang-Mills theory, a similar RG flow equation will provide information on the roles of $k$ and $T$ on the running of the gauge coupling constant. The flow of the theory at $T=0$ has been worked out [6]. It would be interesting to investigate the effect of $T$ on 
such a theory which is known to exhibit asymptotic freedom at $T=0$. If $T$ and $k$ can generate opposite effects as for the scalar theory, there will be nontrivial consequences on the picture of deconfinement transition of quarks and gluons. In addition, the resummation of "hot thermal" loops using this RG approach will readily yield the gauge-independent gluon damping rate and be compared with that obtained in [4] via an effective action. Works along these directions are currently in progress.

1. S. Weinberg, Phys. Rev. D9 (1974) 3357.

2. L. Dolan and R. Jackiw, Phys. Rev. D9 (1974) 3320.

3. E. Braaten and R. D. Pisarski, Phys. Rev. Lett. 64 (1990) 1338; Nucl. Phys. B337 (1990) 569.

4. S.-B. Liao and M. T. Strickland, DUKE-TH-95-81, submitted to Phys. Rev. D.

5. N. Tetradis and C. Wetterich, Int. J. Mod. Phys. A9 (1994) 4029; Nucl. Phys. B422 (1994) 541 and B398 (1993) 659, and references therein.

6. S.-B. Liao and J. Polonyi, DUKE-TH-94-65.

\title{
3.2 Symmetry-Preserving Regularization
}

\author{
S.-B. Liao $[1]$
}

An essential step in identifying the physical contents of quantum field theory is the removal of ultraviolet divergences which arise due to the presence of interactions. The procedure, known as renormalization, operates on the use of some regularization schemes to control the infinites followed by a redefinition of parameters contained in the original lagrangian in such a way that the physical quantities are independent of the choice of regularizations. Although various regularization methods are available, it is often crucial to employ a procedure which respects all the symmetry properties present in the original theory. For example, when studying gauge theories such as QCD or QED, a sharp momentum cut-off regulator would not be appropriate since it explicitly violates gauge invariance.

However, it is possible to introduce the momentum cut-off scale in a symmetry-preserving manner using the so-called "operator cut-off regularization" technique [2]. The method originates from Schwinger's proper-time parameterization [3] with the construction of a smooth smearing function $\rho(\Lambda, s)$ for the proper-time variable $s$, where $\Lambda$ is the usual ultraviolet regulator in the momentum cut-off scheme. Embedding $\rho(\Lambda, s)$ in the $s$ integration, the one-loop contribution to the effective action can be evaluated as:

$$
\left.\operatorname{Tr} \ln \left(\frac{\mathcal{H}}{\mathcal{H}_{0}}\right)\right|_{\text {reg. }}=-\int_{0}^{\infty} \frac{d s}{s} \rho(\Lambda, s) \operatorname{Tr}\left(e^{-\mathcal{H} s}-e^{-\mathcal{H}_{0} s}\right),
$$

for any arbitrary fluctuation operator $\mathcal{H}$. With a suitable choice of $\rho(\Lambda, s)$, the conventional cut-off result is recovered. 
Operator cut-off regularization can also be used to regulate infrared singularities for arbitrary dimension with a generalized smearing function $\rho_{k}^{(d)}(s, \Lambda)$, where $k$ is the lower infrared cut-off. By requiring that both momemtum cut-off and operator cut-off regulators yield the same renormalization group equation, one is lead to

$$
\rho_{k}^{(d)}(s, \Lambda)=\rho^{(d)}\left(\Lambda^{2} s\right)-\rho^{(d)}\left(k^{2} s\right)=\frac{2 s^{d / 2}}{\Gamma(d / 2)} \int_{k}^{\Lambda} d z z^{d-1} e^{-z^{2} s} .
$$

The importance of operator cut-off regularization is that it preserves full symmetries, even gauge symmetry, of the original lagrangian. The scheme merely transfers the divergent structures arising from the integration over space-time to the integration of proper-time parameter $s$, which is independent of symmetry transformation on the background fields. The use of $\rho_{k}^{(d)}(s, \Lambda)$ is solely for the purpose of "technical" convenience to cope with the singularity in the $s$ integration. Possible violation of symmetry can occur only if the smearing function $\rho_{k}^{(d)}(\Lambda, s)$ depends on certain parameters such as $\Phi$ and $p$.

1. S.-B. Liao, DUKE-TH-94-80.

2. M. Oleszczuk, Z. Phys. C64, 533 (1994).

3. J. Schwinger, Phys. Rev. 82, 664 (1951).

\subsection{An Effective Field Theory Approach to the Cosmological Constant Problem}

\section{S. R. Beane}

The problem of the cosmological constant is more acute today than ever before [1]. Recent measurements of the Hubble constant suggest that a non-zero vacuum energy density must be resuscitated in order to avoid conflict with the inflationary paradigm and its associated successes. In particular, cold dark matter models with $\Omega_{\mathrm{VAC}} \simeq 0.8(\Omega=1)$ are consistent with a wide variety of observations. For example, these models reconcile the recent measurement of the extragalactic distance scale with estimated ages of galactic globular structures. Pierce and collaborators [2] have extracted a Hubble constant of $H_{0}=87 \pm 7 \mathrm{~km} \mathrm{~s}^{-1} \mathrm{Mpc}^{-1}$, which is conflict with the age estimate of $16.5 \pm 2 \mathrm{Gyr}$ [3], if one works in a flat universe with no cosmological constant. If one allows both matter and a positive cosmological constant $\left(\Omega_{\mathrm{VAC}}+\Omega_{\mathrm{MATTER}}=1\right)$, these measurements are consistent if $\Omega_{\mathrm{VAC}} \simeq(0.8-0.9)$ (see Figure 1)).

In an application of the effective field theory method, the viewpoint that a small, or vanishing, cosmological constant should follow from the action of a symmetry group at very low energies was investigated [4]. Non-linearly realized Weyl invariance was considered in some detail, with focus on an Euler-Heisenberg effective theory with anomalous Weyl symmetry. A special class of dilaton potentials was shown to possess an interesting reparametrization invariance -or pseudo-symmetry-which might explain why the cosmological constant is so 


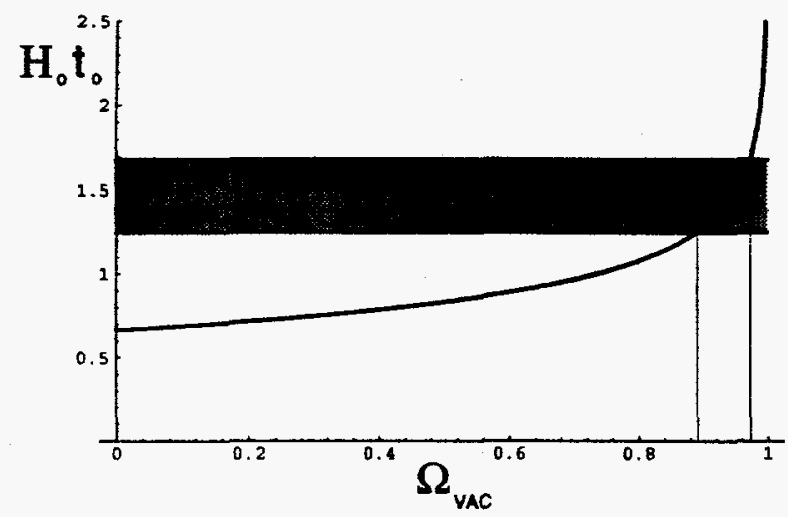

Figure 1: Plot of $H_{0} t_{0}$ vs. $\Omega_{\mathrm{VAC}}$. The curve is the theoretical prediction for a flat Friedmann-Robertson-Walker spacetime with $\Omega_{\mathrm{VAC}}+\Omega_{\mathrm{MATTER}}=1$. The shaded band corresponds to the measured quantities: $H_{0}=87 \pm 7 \mathrm{~km} \mathrm{~s}^{-1} \mathrm{M} \mathrm{pc}^{-1}$ and $t_{0}=16.5 \pm 2 \mathrm{Gyr}$.

small. It was argued that, in a sense, this reparametrization invariance is an exact classical symmetry of superstrings. That this pseudo-symmetry survives at low-energies is not at odds with model-independent characteristics of string-dilaton potentials. Simple dimensional arguments based on this scenario have interesting implications, which can be summarized as:

$$
\rho_{\mathrm{VAC}} \simeq\left(m_{\phi}\right)^{4} \simeq\left(\frac{m_{\mathrm{SUSY}}^{2}}{M_{p}}\right)^{4} \simeq\left(10^{-12} \mathrm{GeV}\right)^{4} \sim \rho_{\mathrm{CRIT}} .
$$

Low-energy supersymmetry breaking $-m_{\mathrm{SUSY}} \simeq 4 \pi v$ - is a consequence of the observational upper bound on $\rho_{\mathrm{VAC}}$. This scenario therefore implies a superstring dilaton with mass $m_{\phi} \simeq$ $10^{-12} \mathrm{GeV}$, giving rise to a new force with range $\lambda \simeq 0.2 \mathrm{~mm}$, and a residual cosmological constant, $\rho_{\mathrm{VAC}} \simeq\left(m_{\phi}\right)^{4} \sim \rho_{\mathrm{CRIT}}$. The observational constraints on a dilaton with this mass, and with couplings of gravitational strength, are not stringent enough to rule out this scenario. Hence, this picture provides a natural way of generating a vacuum energy density of the right size to dominate the energy density of the universe, as is required in the "best-fit" cosmological models discussed above.

1. See, for example, L.M. Krauss and M.S. Turner, FERMILAB-Pub-95/063-A, astro-ph/9504003.

2. M.J. Pierce et al, Nature 371, 385 (1994).

3. D.A. VandenBergh, The Formation and Evolution of Star Clusters, edited by K. Janes, (Astr. Soc. Pacif., San Francisco, 1991).

4. S.R. Beane, DUKE-TH-95-84, hep-ph/9502226. 


\section{A Publications}

\section{A.1 Books}

1. Chaos and Gauge Field Theories, T. S. Biró, S. Matinyan, and B. Müller, World Scientific Publishing Co. (1995).

\section{A.2 Published Articles}

1. Larger Domains of Disoriented Chiral Condensate Through Annealing, Sean Gavin and B. Müller, Phys. Lett. B329, 486-492 (1994).

2. Pair Production in the Quantum Boltzmann Equation, Jochen Rau, Phys. Rev. D50 6911-6916 (1994).

3. Parton Equilibration at RHIC and LHC, T. S. Biró, B. Müller, M. H. Thoma, and X. N. Wang, Nucl. Phys. A566, 543c-546c (1994).

4. Wigner Functional Approach to Quantum Field Dynamics, Stanisław Mrówczyński and B. Müller, Phys. Rev. D50 7542-7552 (1994).

5. Vacuum Fluctuations and Symmetry Breaking in Spaces With Nontrivial Topology, C. Villarreal, Phys. Rev. D51, 2959 (1995).

6. Thermal Photons and Dileptons from Non-Equilibrium Quark-Gluon Plasma, M. Strickland, Phys. Lett. B331, 245-250 (1994).

7. QCD Evolution Equations for High Energy Partons in Nuclear Matter, K. Geiger, B. Müller, Phys. Rev. D50 337-357 (1994).

8. Multiply Strange Nuclear Systems, J. Schaffner, C. B. Dover, A. Gal, C. Greiner, D. J. Millener and H. Stöcker, Ann. Phys. 235, 35-76 (1994).

9. Renormalization Group and Universality, S.-B. Liao and J. Polonyi, Phys. Rev. D51, 4474 (1995).

10. Quantum and Thermal Fluctuations in Field Theory, S.-B. Liao, J. Polonyi and D. Xu, Phys. Rev. D51, 748-764 (1995).

11. Physics and Signatures of the Quark-Gluon Plasma, B. Müller, Rep. Prog. Phys. 28, 1-26 (1995).

12. Lyapunov Exponent and Plasmon Damping Rate in Nonabelian Gauge Theories, T. S. Biró, C. Gong and B. Müller, Phys. Rev. D (in print).

13. Quark Pair Production in a Rapid Chiral Phase Transition, C. Greiner, Z. Phys. A (in print). 


\section{A.3 Submitted Articles}

1. Pion Fusion in Peripheral $p p$ Collisions, A. J. Schramm and B. Müller, DUKE-TH-9467, submitted to Phys. Rev. D.

2. Open Charm Production in an Equilibrating Parton Plasma, P. Lévai, B. Müller, X.-N. Wang, DUKE-TH-95-83, submitted to Phys. Rev. D.

3. Renormalization Group Approach to Field Theory at Finite Temperature, S.-B. Liao and M. T. Strickland, DUKE-TH-95-81, submitted to Phys. Rev. D.

4. Derivative Expansion and Renormalization Group, S.-B. Liao and C. Gong, DUKETH-94-63, submitted to

5. Renormalization Group for Yang-Mills Theories, S.-B. Liao and J. Polonyi, DUKETH-94-65.

6. Meson-Nucleon Couplings for Weak Decays of Hypernuclei, M. Savage and R. Springer, DUKE-TH-94-77.

7. On Connection between Momentum Cut-Off and Proper-Time Regulations, S.-B. Liao, DUKE-TH-94-80, submitted to Phys. Rev. D.

8. Wavepacket Dynamics in SU(2) Yang-Mills Theory, C. R. Hu, S. G. Matinyan, B. Müller, A. Trayanov, T. M. Gould, S. D. Hsu, E. R. Poppitz, DUKE-TH-95-85, submitted to Phys. Rev. D.

9. Dynamics of Soft Modes in Thermal Gauge Field Theories, T. S. Biró, S. Matinyan, and B. Müller, DUKE-TH-95-90, submitted to Phys. Rev. Lett.

10. Neutral Pion Photoproduction on Light Nuclei in Baryon Chiral Perturbation Theory, S. Beane, C. Y. Lee and U. van Kolck, DUKE-TH-95-88. Submitted to Phys. Rev. C.

11. Can Reparametrization Invariance Solve the Cosmological Constant Problem?, S. R. Beane, DUKE-TH-95-84. Submitted to Phys. Lett. B.

12. From Reversible quantum Microdynamics to Irreversible Quantum Transport, J. Rau and B. Müller, DUKE-TH-95-92. Submitted to Physics Reports.

\section{A.4 Conference Reports}

1. X. N. Wang, B. Müller, D-Meson as a Probe of Early Parton Rescattering, Nucl. Phys. A566, 555c-558c (1994).

2. B. Müller, Relativistic Heavy Ion Collisions: Probing the Early Universe in the Laboratory, Proceedings of the Workshop on Pre-Equilibrium Parton Dynamics, LBL, Berkeley, August 1993, p. 1-39. Publication LBL-34831/CONF-9308181. 
3. B. Müller, Colored Chaos, NATO Advanced Research Workshop, Hot Hadronic Matter, Divonne, June 1994. To be published by Plenum Press.

4. B. Müller, Chaos in Nonabelian Gauge Theories, Workshop on Quantum Infrared Physics, Paris, June 1994. To be published by World Scientific.

5. S.-B. Liao, Renormalization Group Approach to Field Theory, Workshop on Quantum Infrared Physics, Paris, June 1994. To be published by World Scientific.

6. R. P. Springer, The Baryon Decuplet in Chiral Perturbation Theory, Proceedings of the Lanczos International Centenary Conference, Raleigh, ed. J. David Brown et al., (SIAM, 1994) p. 611.

7. B. Müller, Quark Matter '95: What Have We Learned and What Needs To Be Done?, to be published in Nucl. Phys. A.

8. B. Müller, The Physics of the Quark-Gluon Plasma, Seventh A. J. Swieca Summer School on Nuclear Physics, Sao Paolo, Brazil (1995).

9. S. G. Matinyan, Classical Chaos and High Energy Collisions, in Quantum-Classics Correspondence, Philadelphia, (1995).

10. C. Greiner, A. Diener, J. Schaffner and H. Stöcker, Strange Matter-A New Domain of Nuclear Physics, 17th Symposium on Nuclear Physics at Oaxtepec, Mexico, 1994, Revista Mexicana de Fisica 40, Suplemento 1 (1994).

11. C. Greiner, A. Diener, J. Schaffner and H. Stöcker, Strange Matter-A New Domain of Nuclear Physics, Proc. of the NATO Advanced Study Institute on 'Hot and Dense Matter' at Bodrum, Turkey, 1993, ed. W. Greiner, H. Stöcker and A. Gallmann, B335 (Plenum Press, 1994).

12. C. Greiner, K. Wagner and P.-G. Reinhard, Finite Memory in the Collision Processes of a Fermionic System and its Effect on Relativistic Heavy Ion Collisions, Proc. of the NATO Advanced Study Institute on 'Hot and Dense Matter', Bodrum, Turkey, B335 (Plenum Press, 1994).

13. Two-Body Bound States and the Bethe-Salpeter Equation, M. Pichowsky and M. T. Strickland, 1994 HUGS at CEBAF Proceedings, (1995).

14. Deuteron Photodisintegration Above Pion Threshold, M. T. Strickland, 1994 HUGS at CEBAF Proceedings, (1995).

15. Branching Processes and Multi-Particle Production, Proceedings of the Fifth Blois Workshop: Elastic and Diffractive Scattering, Brown University, Ed. H. Fried, K. Kang, and C.-I. Tan,(1994), p. 225. 
16. C. Greiner, Physics of Strange Matter for Relativistic Heavy Ion Collisions, QuarkGluon Plasma II, ed. R. Hwa, Advanced Series on Directions in High Energy Physics, World Scientific.

17. C. Speiles, C. Greiner, H. Stöcker and J. P. Coffin, Distillation of Strangelets for Low Initial $\mu / T$, submitted to ??.

18. C. Greiner, Strange Matter Searches under the Lamppost, Proc. of the Symposium on 'Strangeness and Quark Matter' Crete, Greece, 1994.

19. C. Greiner, Physics in the Baryon-Rich Regime, Proc. 'Quark Matter '95, Monterey, CA (to be published in Nucl. Phys. A.).

\section{B Lecture Activities}

\section{B.1 Invited Talks}

1. B. Müller, Probing Fundamental Laws with the Hydrogen Lamb Shift, QED-Symposium, Tokyo Metropolitan University, Tokyo, Japan, July 11, 1994.

2. B. Müller, Four Lectures on Quark Gluon Plasma and Relativistic Heavy Ion Collisions, Tokyo Metropolitan University, July 12-20, 1994.

3. B. Müller, Chaos in Nonabelian Gauge Theories, Workshop on Quantum Infrared Physics, American University of Paris, France, June 10, 1994.

4. B. Müller, Colored Chaos, NATO Advanced Research Workshop on Hot Hadronic Matter, Divonne-les-Bains, France, June 28, 1994.

5. B. Müller, Four Lectures on Relativistic Heavy Ion Physics, Hungarian School on Heavy Ion Physics, Budapest, Hungary, August 16-28, 1994.

6. S. G. Matinyan, Classical Chaos and High Energy Collisions, in Proceedings, "QuantumClassics Correspondence", Philadelphia, September 1994.

7. B. Müller, Chaos in Nonabelian Gauge Theory, Workshop on Parton Production and Transport, ECT*, Trento, Italy, October 2-14, 1994.

8. S. G. Matinyan, Quark Degrees of Freedom and Total Photoabsorption in Nuclei, TUNL-FELL Workshop on the Development of a Polarized Gamma-Ray Beam, December 1994, Durham, NC.

9. S. Beane, Chiral Symmetry and Few-Body Nuclear Reactions, TUNL-FEL Workshop, Durham, December 1994. 
10. C. Greiner, Quark Pair Production in a Rapid Chiral Phase Transition, Quark Matter '95 Conference in Monterey, CA., January 1995.

11. B. Müller, Quark Matter '95: What Have We Learned and What Needs to be Done? Opening Lecture, Quark Matter '95 Conference, Monterey CA, January 9-13, 1995.

12. C. Greiner, Strangeness Production, STAR Physics Workshop at Monterey, CA, January 15, 1995.

13. B. Müller, Five Lectures on Relativistic Heavy Ion Collisions and Quark Gluon Plasma, A. J. Swieca Summer School on Nuclear Physics, Campos do Jordao, Sao Paolo, Brazil, January 22 - February 4, 1995.

14. R. P. Springer, Effective Theories for Nucleons, University of Wisconsin, Madison, February 10, 1995.

15. R. P. Springer, The Baryon Decuplet in Chiral Perturbation Theory, Ohio State University, February 13, 1995.

16. S.-B. Liao, Effective Field Theory and Renormalization Group, Ninth Spring School on Particles and Fields, Taichung, Taiwan, March 24, 1995.

17. S. Beane, Neutral Pion Photoproduction on Nuclei in Baryon Chiral Perturbation Theory, Workshop on Chiral Dynamics in Hadrons and Nuclei, Seattle, May 1995.

\section{B.2 Contributed Talks and Seminars}

1. S.-B. Liao, Renormalization Group and Universality, National Tsing-Hua University, Hsinchu, Taiwan, May 15, 1994.

2. S.-B. Liao, Renormalization Group and Gauge Symmetry, National Chiao-Tung University, Hsinchu, Taiwan, May 19, 1994.

3. S.-B. Liao, Renormalization Group and Universality, National Chung-Hsing University, Taichung, Taiwan, May 20, 1994.

4. B. Müller, Relativistic Heavy Ion Collisions and Quark-Gluon Plasma, Physics Colloquium, Technical University Dresden, Germany, June 21, 1994.

5. B. Müller, Parton Cascade Model of Relativistic Heavy Ion Collisions, Niels-Bohr Institute, Copenhagen, Denmark, June 23, 1994.

6. Michael Strickland, Making the Transition from QCD to Mesons, 1994 HUGS Summer School at CEBAF, June 29, 1994.

7. B. Müller, Chaos in Nonabelian Gauge Theories, Yukawa Institute, University of Kyoto, Japan, July, 13, 1994. 
8. B. Müller, Disoriented Chiral Condensates, Physics Department, University of Kyoto, Japan, July 13, 1994.

9. C. Greiner, Quark Pair Production in a Rapid Chiral Phase Transition, Universität Erlangen, July 14, 1994.

10. B. Müller, Disoriented Chiral Condensates, University of Tsukuba, Japan, July 20, 1994.

11. B. Müller, Chaotic Dynamics of Nonabelian Gauge Fields, University of Tsukuba, Japan, July 21, 1994.

12. B. Müller, QCD Description of Relativistic Heavy Ion Collisions, Physics Department, University of Tokyo, July 24, 1994.

13. C. Greiner, Quark Pair Production in a Rapid Chiral Phase Transition, Universität Giessen, December 22, 1994.

14. S.-B. Liao, Renormalization Group and Field Theory at Finite Temperature, University of North Carolina, Chapel Hill, February 9, 1995.

15. S.-B. Liao, Renormalization Group and Field Theory at Finite Temperature, National Taiwan University, Taipei, Taiwan, February 27, 1995.

16. S.-B. Liao, On Applications of Renormalization Group in Quantum and Statistical Field Theory, National Cheng-Kung University, Tainan, Taiwan, March 2, 1995.

17. S.-B. Liao, On Applications of Renormalization Group in Quantum and Statistical Field Theory, National Tsing-Hsu University, Hsinchu, Taiwan, March 6, 1995.

18. S.-B. Liao, Renormalization Group and Field Theory at Finite Temperature, National Tsing-Hsu University, Hsinchu, Taiwan, March 6, 1995.

19. C. Greiner, Strange Matter, Lawrence Berkeley Laboratory, Berkeley, CA., March 8, 1995.

20. C. Greiner, Memory Effects in Relativistic Heavy Ion Collisions, Theory Seminar at the Lawrence Berkeley Laboratory, Berkeley, CA., March 9, 1995.

21. S.-B. Liao, On Applications of Renormalization Group in Quantum and Statistical Field Theory, National Chung-Cheng University, Chia-yi, Taiwan, March 9, 1995.

22. S.-B. Liao, On applications of Renormalization Group in Quantum and Statistical Field Theory, National Chung-Hsing University, Taichung, Taiwan, March 10, 1995.

23. B. Müller, Colored Chaos, Physics Colloquium, Kent State University, March 10, 1995.

24. B. Müller, Colored Chaos, University of Washington, Seattle, March 15, 1995. 
25. S.-B. Liao, On Applications of Renormalization Group in Quantum and Statistical Field Theory, Tamkang University, Tamsui, Taiwan, March 15, 1995.

26. S.-B. Liao, Renormalization Group and Field Theory at Finite Temperature, Academia Sinica, Taipei, Taiwan, March 17, 1995.

27. S.-B. Liao, On Applications of Renormalization Group in Quantum and Statistical Field Theory, National Chiao-Tung University, Hsinchu, Taiwan, March 31, 1995.

28. S. G. Matinyan, Wavepacket Dynamics in Yang-Mills Theory, Kentucky University, Lexington, April 3, 1995.

29. B. Müller, Colored Chaos, University of North Carolina, Chapel Hill, April 6, 1995.

30. B. Müller, Colored Chaos, University of Virginia, Charlottesville, April 10, 1995. 


\section{Triangle Nuclear Colloquium Series}

9/8 Jonathan Engel (University of North Carolina)

Microscopic T-Violating Optical Potential

9/20 Brian Serot (University of Indiana)

Nuclear Structure Constraints on Chiral Hadronic Field Theories

9/27 Harry Lee (Argonne National Laboratory)

Chiral symmetry and Hadronic and Electromagnetic Production

of Threshold Pions

9/30 Mahir Hussein (Sao Paulo and MIT)

Parity and Time Reversal Symmetry Breaking

in Low Energy Neutron-Nucleus Scattering

10/25 Rudy Hwa (University of Oregon)

Scaling Properties of Quark-Hadron Phase Transition

11/1 Ted Barnes (Oak Ridge National Laboratory)

Two Photon Physics and Meson Spectroscopy

11/8 Matthias Burkardt (University of Washington, Seattle)

A Small Transverse Lattice and the Deuteron

11/15 Barry Holstein (University of Massachusetts)

Polarizability and Hadron Structure

11/17 Alexander Shebeko (Kharkov)

Polarization Phenomena in Photo- and Electro-Disintegration

of Lightest Nuclei

11/29 Leonard Kisslinger (Carnegie Mellon University)

The Parity-Violating Pion-Nucleon Interaction:

Why Is It Weaker than Weak?

12/6 Xiangdong Ji (Massachusetts Institute of Technology)

Resonances, Higher Twists, and Sum Rules 
12/13 George Bertsch (University of Washington)

Pion Interferometry in RHIC

2/7 David London (University of Montreal)

Determining the Kobayashi-Maskawa Matrix

2/14 Keh-Fei Liu (University of Kentucky)

Lattice Calculations of Nucleon Structure

2/21 Eric Braaten (Northwestern University)

Finite Temperature Gauge Theories

2/28 Franco Iachello (Yale University)

The Role of Dynamic Symmetries and Supersymmetries in Nuclear Physics

3/14 Brad Keister (National Science Foundation)

Compton Scattering from the Nucleon

3/28 Mikkel Johnson (Los Alamos National Laboratory)

Hadrons in Nuclei and QCD Sum Rules

4/4 Michael Strayer (Oak Ridge National Laboratory)

The First Fifty Years of Computational Physics

4/11 Miklos Gyulassy (Columbia University)

Boiling the Nuclear Glue at RHIC

4/25 Witold Nazarewicz (Oak Ridge National Laboratory)

The Structure of Exotic Nuclei

$5 / 2 \quad$ Russell Betts (Argonne National Laboratory)

The APEX Experiment 


\section{Theory Seminars at Duke}

5/25 Carsten Greiner (Duke University)

Quark Pair Production in the Chiral Phase Transition

5/27 Klaus Kinder-Geiger (CERN, Theory Division)

QCD Based Space-Time Description of Heavy Ion Physics

5/30 Raju Venugopalan (University of Minnesota)

The Color Field of a Large Nucleus at Small $x$

5/31 Walter Greiner (University of Frankfurt, Germany)

News about Cluster Radioactivity and Cold Fission

6/2 Walter Greiner (University of Frankfurt, Germany)

What We Know and Don't Know about the Quark-Gluon-Plasma

9/21 Brian Serot (Indiana University)

Effective Relativistic Theories of the Nuclear Force

9/28 Dirk Rischke (Columbia University)

Perturbative vs. Lattice QCD Energy Density Correlators at High Temperature

9/29 Charles Kane (University of Pennsylvania)

Edge States in the Quantum Hall Effect

9/30 Dirk Rischke (Columbia University)

Relativistic Hydrodynamics

9/14 Silas Beane (Duke University)

Neutral Pion Photoproduction on Nuclei in Chiral Perturbation Theory.

10/3 Roxanne Springer (Duke University)

$C P$ Violation in the B System

10/3 Martin Greiner (Universität Giessen)

A New Tool to Study Selfsimilarity and Clustering in Complex Reactions

10/24 Sean Fleming (Northwestern University)

$J / \psi$ and $\psi^{\prime}$ Production at the Tevatron

11/2 Ted Barnes (University of Tennessee)

New Results from the Chain Gang 
11/9 Y. Jack $\mathrm{Ng}$ (University of North Carolina)

Limitation on the Quantum Measurement of Space-Time Distances

11/16 Jane Richardson (Duke University)

Designing New Proteins

11/30 Bruce Carney (University of North Carolina)

Astronomy and Astrophysics at UNC-Chapel Hill

12/7 Jane (and David) Richardson (Duke University)

Designing New Proteins: the Sequel

1/18 Patrick O'Shea (Duke University)

Some Issues in Beam Physics

1/30 Sergei Matinyan (Duke University/Yerevan, Armenia)

Charge Symmetry and Charge Independence: $Q C D$ View

2/15 Eugen Merzbacher (University of North Carolina)

News from the APS Task Force on Journal Growth

2/20 Eric Braaten (Northwestern University)

Charm Production at the Tevatron

2/20 Max Lohe (University of Texas, Austin/University Darwin)

Tensor Operators in Quantum Groups

2/23 Roxanne Springer (Duke University)

KNN Couplings for Hypernuclei Decay

3/1 Henry Weller (Duke University)

The TUNL-FELL Inverse Compton Scattering Project

3/9 Silas Beane (Duke University)

Reparametrization Invariance and the Cosmological Constant Problem

4/24 Klaus Kinder-Geiger (CERN)

A Quantum Kinetic Approach to Non-Equilibrium QCD Processes at High Energies

4/24 Martin Olsson (University of Wisconsin at Madison)

The Flux Tube Model of Mesons 